\title{
STUDY OF THE POST-FLARE LOOPS ON 29 JULY 1973
}

\author{
IV. Revision of $T$ and $n_{e}$ Values and Comparison with the Flare of 21 May 1980
}

\author{
Z. ŠVESTKA ${ }^{\text {I }}$ H. W. DODSON-PRINCE ${ }^{2}$, S. F. MARTIN ${ }^{3}$, O. C. MOHLER ${ }^{2}$, \\ R. L. MOORE ${ }^{4}$, \\ J. T. NOLTE ${ }^{5}$, and R. D. PETRASSO ${ }^{5}$
}

(Received 12 May; in revised form 18 September, 1981)

\begin{abstract}
We present revised values of temperature and density for the flare loops of 29 July 1973 and compare the revised parameters with those obtained aboard the SMM for the two-ribbon flare of 21 May 1980. The 21 May flare occurred in a developed sunspot group; the 29 July event was a spotless two-ribbon flare. We find that the loops in the spotless flare extended higher (by a factor of 1.4-2.2), were less dense (by a factor of 5 or more in the first hour of development), were generally hotter, and the whole loop system decayed much slower than in the spotted flare (i.e. staying at higher temperature for a longer time). We also align the hot X-ray loops of the 29 July flare with the bright $\mathrm{H} \alpha$ ribbons and show that the $\mathrm{H} \alpha$ emission is brightest at the places where the spatial density of the hot elementary loops is enhanced.
\end{abstract}

\section{Introduction}

The two-ribbon flare of 29 July 1973, observed in soft X-rays on Skylab and in the $\mathrm{H} \alpha$ light on the ground, was the subject of a detailed study by Moore et al. (1979) during the Skylab Flare Workshop (Sturrock, 1979). However, some measurements and their analyses could not be completed before the deadline set by the editor of the Workshop Proceedings so that quite a few results published there had to be of a preliminary nature. Therefore, a series of completed and partly revised studies of this flare event has been published in Solar Physics, starting with a discussion of the dynamics of the X-ray loops by Nolte et al. (1979, referred to as Paper I); next, Petrasso et al. (1979, Paper II) have analyzed the physical parameters in the X-ray loops, and Martin (1979, Paper III) has discussed the dynamics of the $\operatorname{cool} \mathrm{H} \alpha$ loops. The present paper (numbered IV) brings a synthesis of all the obtained results, revises temperature and density, and compares the resulting data with observations of another event of this kind, observed aboard the SMM on 21 May 1980.

In order to save space, we will refer to the three preceding papers and Moore et al. (1979) rather than repeat what already has been said there. Such reference will also be used for some figures and tables published in the previous studies.

1 Space Research Laboratory, Utrecht, The Netherlands, and Center for Astrophysics and Space Science, University of California, San Diego, La Jolla, U.S.A.

2 McMath-Hulbert Observatory, University of Michigan, U.S.A.

3 San Fernando Observatory of California State University, Northridge, U.S.A.; presently at Big Bear Solar Observatory, Caltech, California, U.S.A.

4 Big Bear Solar Observatory, Caltech, California, U.S.A.; presently at the Marshall Space Flight Center, Huntsville, Alabama, U.S.A.

5 American Science and Engineering, Inc., Cambridge, Mass., U.S.A. 


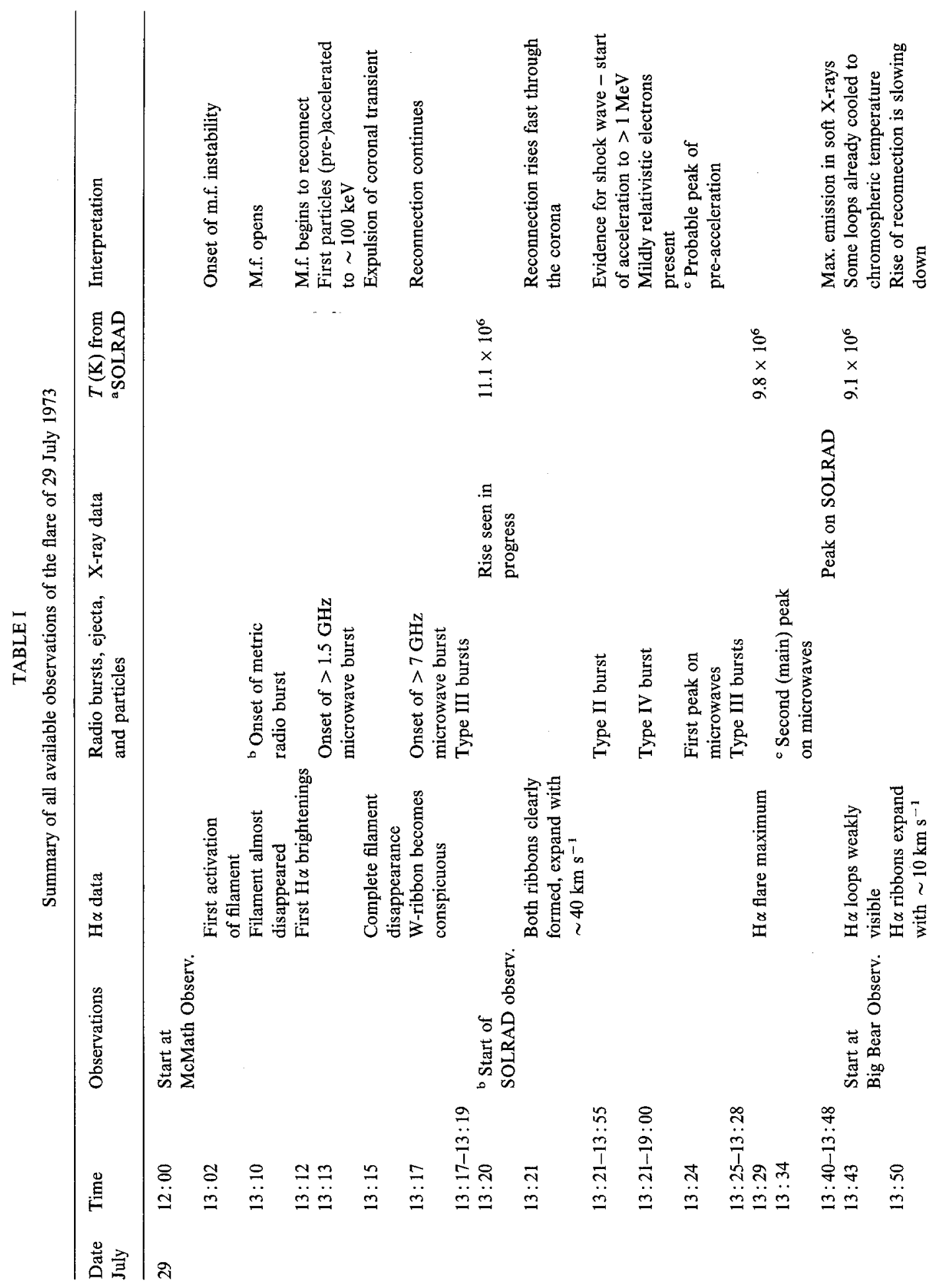




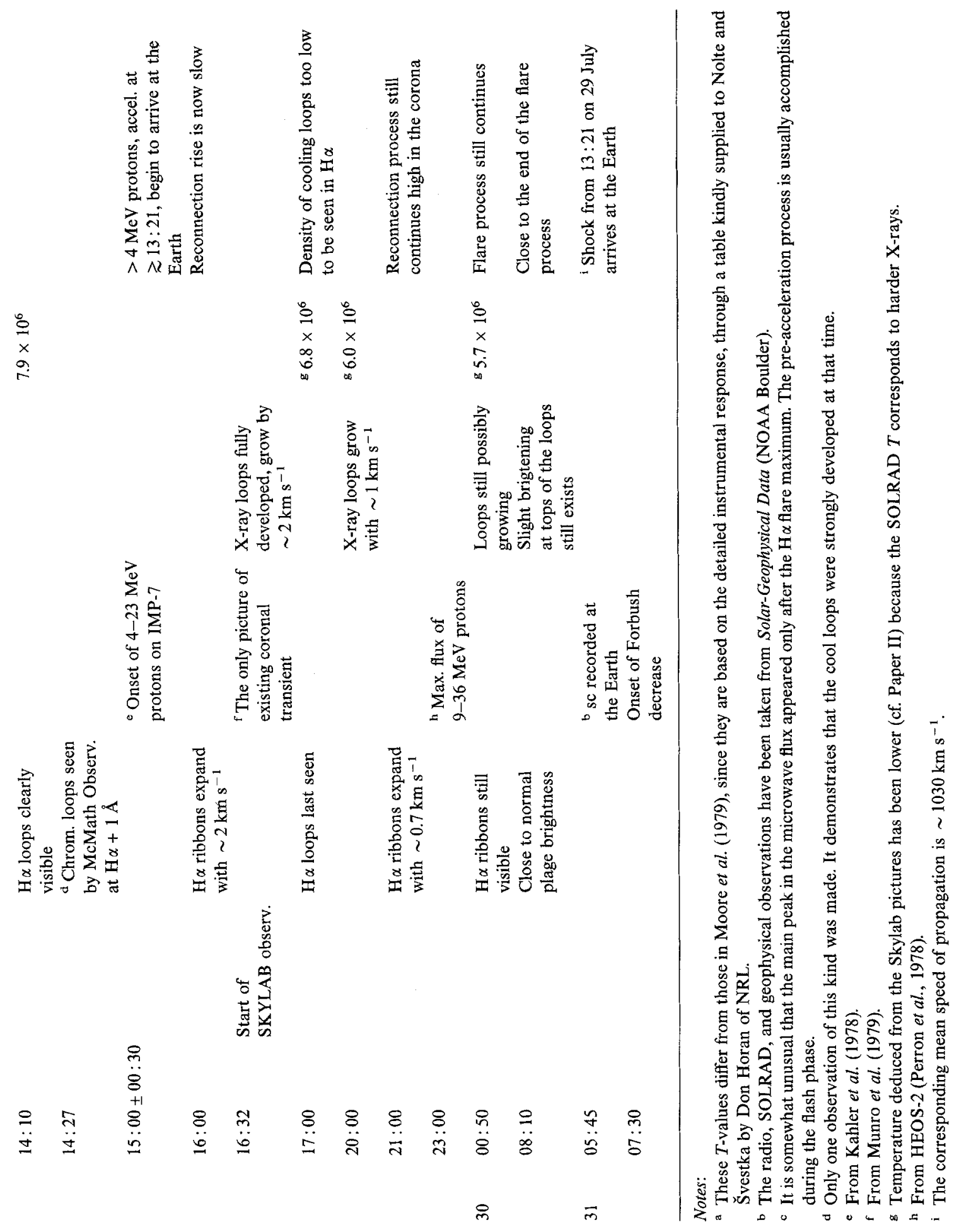




\section{Available Observations of the July 29, 1973 Event}

Table I illustrates the whole development of the flare, the sequence of its observations on the ground and in space, the associated radio events and ejecta, electron temperature as deduced from Skylab data (by comparing 1-8 $\AA$ with 8-20 $\AA$ flux), and suggested interpretations of the observed phenomena. The table is self explanatory.

One can see that the flare was covered completely in the $\mathrm{H} \alpha$ line at the McMathHulbert Observatory in Michigan and, from the time of the soft X-ray maximum, also with high spatial resolution at the Big Bear Observatory in California. The AS \& E (S-054) soft X-ray pictures from Skylab represent a unique set of observations, because no other system of hot 'post-flare' loops has been observed so far with such a high spatial resolution. The pictures, taken through two different $\mathrm{X}$-ray filters, make it possible to establish the spatial distribution of temperature and density throughout the loop system, as well as the time variation of these quantities. Thus, the whole set of observations is uniquely adequate for obtaining detailed quantitative data about physical conditions in the 'post-flare' loops. It is a pity, of course, that the Skylab observations started only late in the flare development so that we have no coronal pictures for the early phase of the flare. Also SOLRAD records (because of night on the SOLRAD satellite) and the high-resolution Big Bear data do not cover the early flare development.

The flare itself might not have been quite a typical representative of the loop-prominence flares, because it occurred in an old spotless region, whereas the strongest known $\mathrm{H} \alpha$ loop prominences have developed inside spot groups. Therefore, we compare the obtained results, wherever it is possible, with another two-ribbon flare observed in X-rays which occurred inside a fully developed active region on 21 May 1980. This flare was observed by the Hard X-Ray Imaging Spectrometer (HXIS) aboard the SMM (for details of the instrument see Van Beek et al., 1980) with a lower spatial resolution (8"), but incomparably better time coverage (Hoyng et al., 1981; Švestka et al., 1982).

\section{The Preflare Situation}

The flare of 29 July 1973 occurred in McMath plage 12461, a center of activity in its third rotation. The fully developed sunspot group, seen one rotation before, disappeared, and on July 26 the region emerged from behind the east limb as an old, extensive but spotless, active region. On the limb, one could see in soft $\mathrm{X}$-rays its extensive loop system extending up to an altitude of $260000 \mathrm{~km}$ above the solar limb (Howard and Švestka, 1977).

As described in Moore et al. (1979) and by Moore and LaBonte (1980), the flare followed the activation and disappearance of a large, dark filament which was embedded in the coronal loops visible in X-rays (Figure 1a; for drawings see Figure 2 in Paper III and Figure 2 in Moore $e t$ al.). Some precursory changes in the active region could be seen as early as 12:23 (Moore and LaBonte, 1980), but an activation of the large filament was recognized first at 13:02. It marked the onset of instability in the magnetic field which eventually led to the filament eruption, supposed opening of an originally closed field, and the onset of coronal and chromospheric heating seen as the flare. 

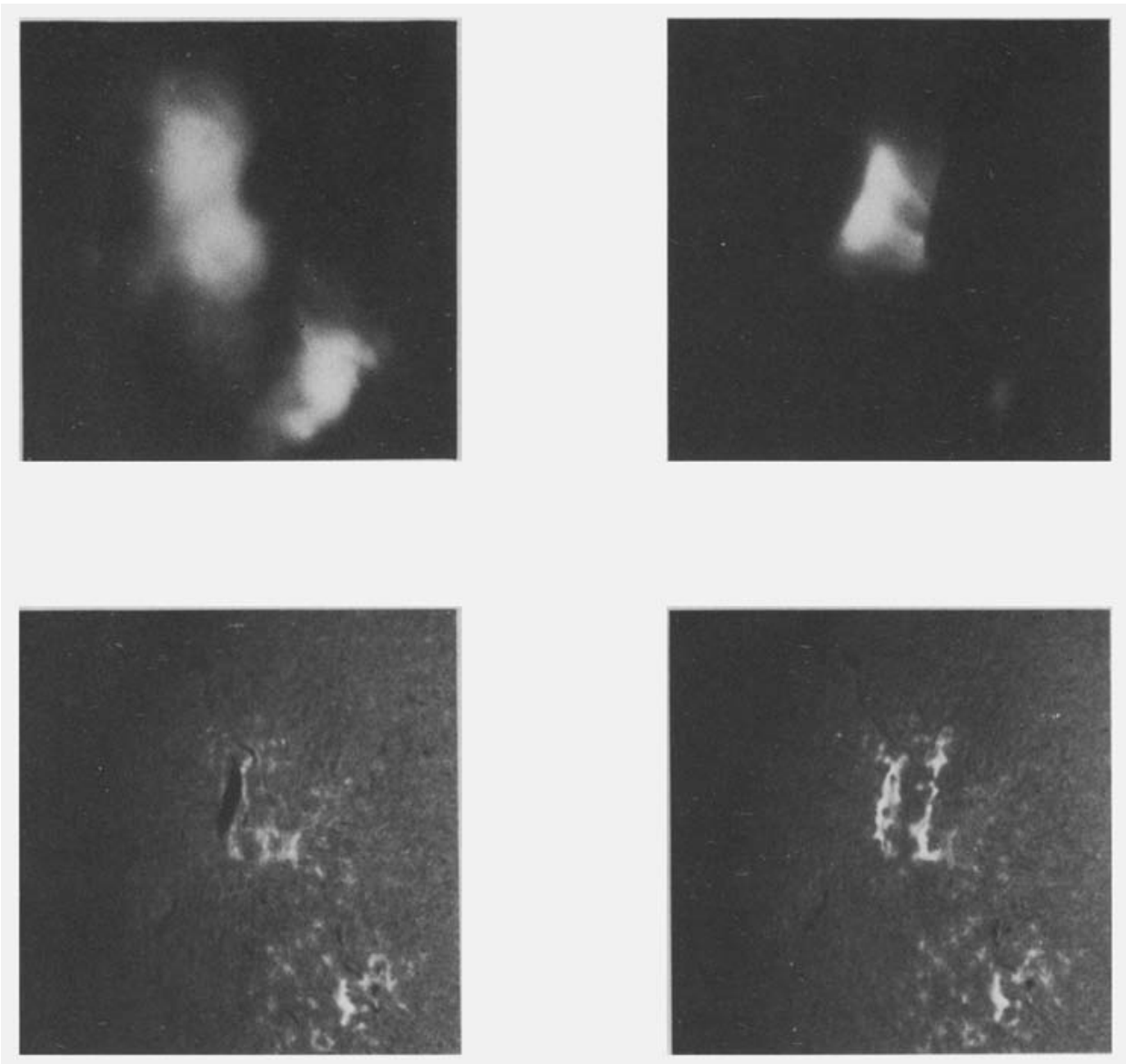

Fig. 1. Soft X-ray pictures (3-54 $\AA$, above) and $\mathrm{H} \alpha$ photographs (below) of the preflare situation (left) and the flare emission at 16:43 on July 29, 1973 (right). The exposure times in X-rays are not identical: $16 \mathrm{~s}$ on the left and $1 \mathrm{~s}$ on the right.

This instability needs a trigger. It has been found before that such a trigger may be a newly emerging flux (Bruzek, 1952; Rust et al., 1975). Also in the case of the flare of 21 May, 1980, whicn we will use for a comparison throughout this paper, a newly emerging flux was detected within $5^{\prime \prime}$ of the point where the pre-flare filament began to break up (Hoyng et al., 1981). Michalitsanos and Kupferman (1974), as well as Howard and Švestka (1977) and Moore and LaBonte (1980) were unable, however, to discover any significant change in the magnetic field where the flare of July 29 occurred. Hence, there is no indication whatsoever that a new flux emerged in the active region in association with this flare.

Another possibility may be that the flare was triggered by a slow-mode-wave disturbance arriving from another active region as is often the case with the disparition brusques (cf. Švestka, 1976, pp. 229-231; Rust and Švestka, 1979; and references therein). We were unable, however, to detect any likely source of such a disturbance: 


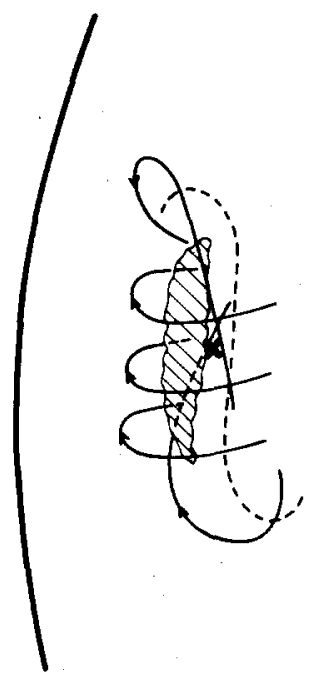

Fig. 2. Sketch of the inferred magnetic field configurations before the filament eruption. The heavy arc is the limb; the dashed line is the photospheric neutral line. Lines with arrow-heads are magnetic field lines, and $\mathrm{X}$ marks the place where reconnection is supposed to occur. (After Moore and LaBonte, 1980).

the McMath 12461 was a member of an activity complex which was quite isolated on the Sun (nearest other region was more than $800000 \mathrm{~km}$ away and no activity preceding this flare was seen in the activity complex itself (Howard and Švestka, 1977)).

Thus one has to suppose that there must have been an internal trigger in the magnetic field configuration itself. A possibility for such a self-induced instability has been recently proposed van Van Tend and Kuperus (1978) by showing that the currents concentrated above a neutral line (and thus associated with the dark filament) have a distinct upper limit. If this limit is reached, the current cannot be stored in the corona any more and an instability develops. Other possible ways by which a preflare filament might be destabilized have been summarized, and some of them further developed, by Priest and Milne (1980). Moore and LaBonte (1980) believe that an untenable shear was the immediate cause of the flare (cf. Figure 2).

\section{Growth of the Loops}

The early development of the flare has been described in detail in Section 8.3.3 of Moore et al. In addition to that description, Moore and LaBonte (1980) have shown that the first traces of the bright flare ribbons could be seen in $\mathrm{H} \alpha$ as soon as the filament began to dissolve. The distance of these embryo ribbons from the $H_{\|}=0$ line was much less than the pre-eruption height of the bottom of the large erupting filament (see Figure 3) which, according to Paper III was $>38000 \mathrm{~km}$; also the first visible loops were much lower (Paper III). If we assume that the whole flare process is a product of sequential field-line reconnection, this means that the reconnection process began below the fila- 
ment, approximately at the time when the filament began to rise and disappear. A configuration of highly sheared field below the filament which might be responsible for the starting reconnection and contemporary destabilization of the filament, is proposed in Figure 2. However, the main phase of the flare did not begin before 13:12, when the large filament had almost disappeared, and it was not until $13: 21$ that both of the $\mathrm{H} \alpha$ ribbons were fully developed.

The growth of the separation distance of the $\mathrm{H} \alpha$ flare ribbons and the evolution of the ribbon width were measured on the McMath-Hulbert filtergrams by Dodson-Prince and on the Big Bear filtergrams by LaBonte. Whereas LaBonte's measurements can be found in Moore et al. (their Figure 8.3a), the results obtained by Dodson-Prince are

(A) Relative Separation of Outer Edges of Bright Ribbons in Central Part of Flore

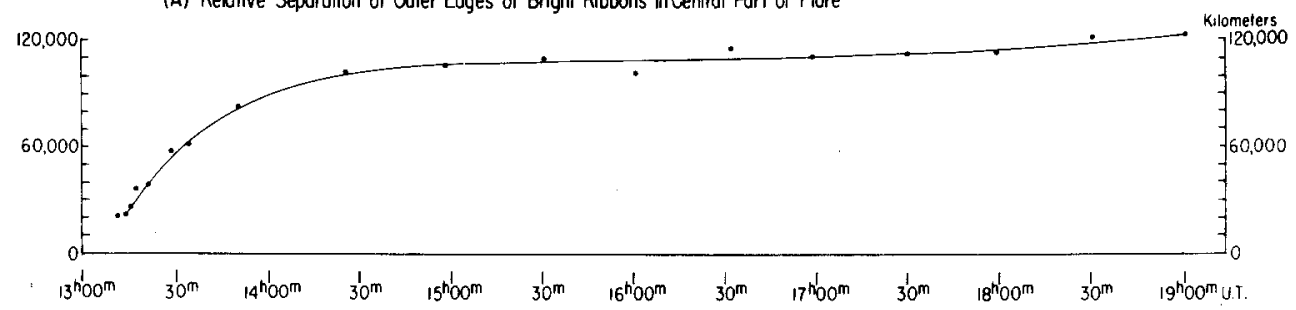

(B) Location of Inner and Outer Edges of Each Bright Ribbon in Central Part of Flare

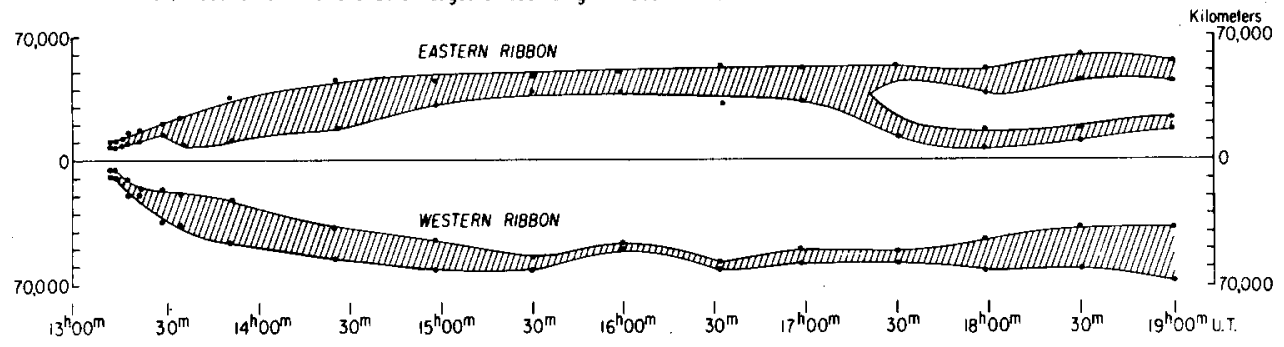

(C) Velocity of Seporotion of Outer Edges of Bright Ribbons in Central Part of Flore

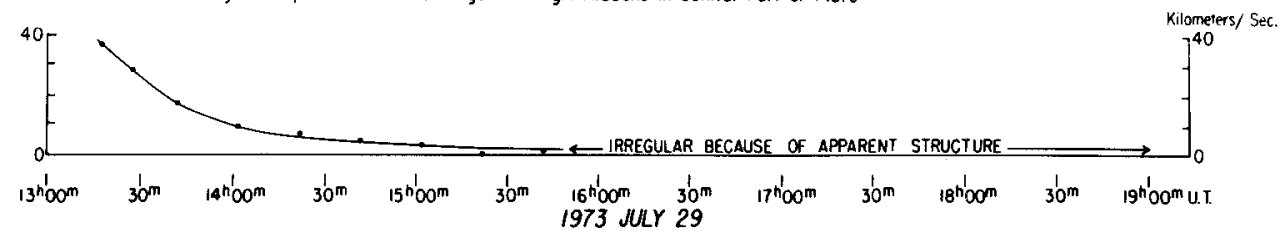

Fig. 3. Separation of the bright $\mathrm{H} \propto$ flare ribbons as measured at the McMath-Hulbert Observatory of the University of Michigan. 
shown in Figure 3. At the start of the flare (which was not seen at Big Bear), the outer edges of the original two bright embryo ribbons were separated by $\sim 20000 \mathrm{~km}$; the inner edges by $\sim 13000 \mathrm{~km}$. In comparison to these, the altitude of the bottom of the large filament prior to its eruption was $>38000$, perhaps as high as $60000 \mathrm{~km}$ (Paper III). The speed of separation of the outer edges of the two ribbons was as high as $\sim 38 \mathrm{~km} \mathrm{~s}^{-1}$ during the first $10 \mathrm{~min}$ of the flare $(13: 12-13: 22)$, and decreased to $\sim 18 \mathrm{~km} \mathrm{~s}^{-1}$ at $13: 42$, when the Big Bear observations started. During the first hour of the flare, each of the ribbons attained widths of the order of $25000 \mathrm{~km}$ in the portion of the flare where Dodson-Prince's measurements were made. The velocities of expansion of the ribbons, and the distances of the inner and outer edges of the ribbons from the $H_{\|}=0$ line, are shown in Figure 8.4 of Moore et al., both from the McMath-Hulbert and Big Bear measurements.

The same figure in Moore et al. also shows the velocities of growth of the $\operatorname{cool}(\mathrm{H} \alpha)$ and hot (X-ray) loops, as measured by Martin ( $\mathrm{H} \alpha)$ and Nolte (X-rays), and Figure 8.5 in Moore et al. shows the resulting altitudes of these two kinds of loops. Because the

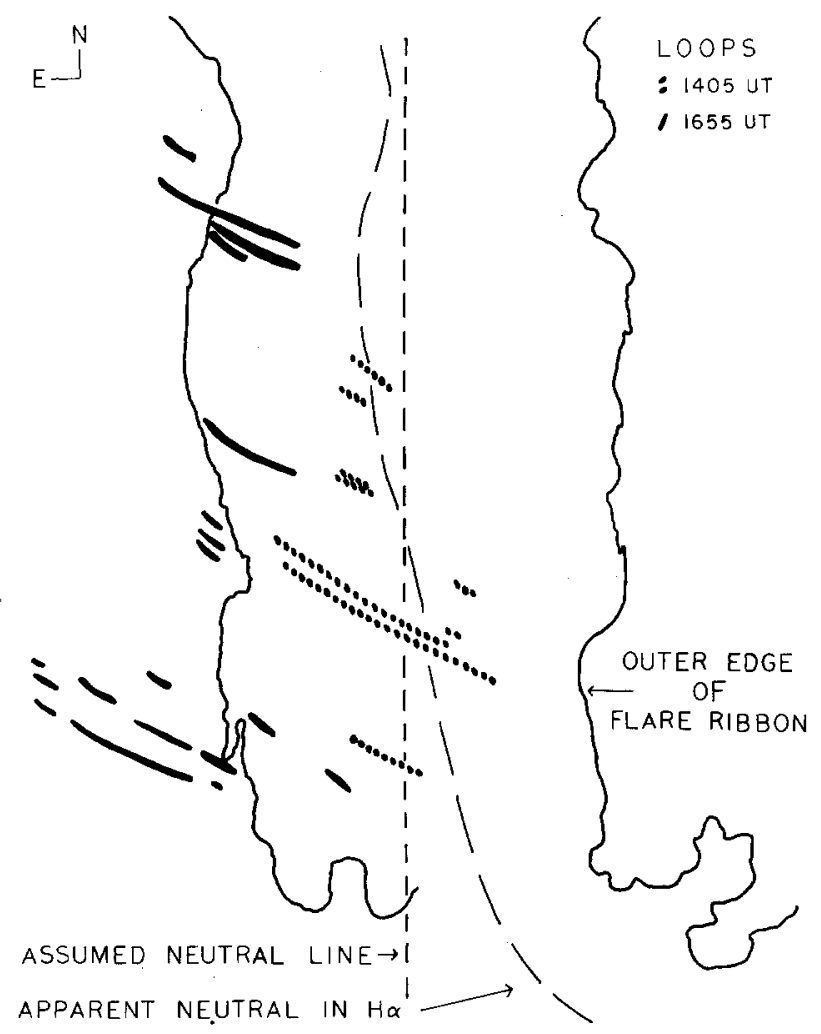

Fig. 4. The $\operatorname{cool}(\mathrm{H} \alpha)$ loops as seen at 14:05 (dotted) and at 16:55 (full lines). This figure explains the differences between Figure 8.5 in Moore et al. and Figure 5 in Paper III. 
loops were seen in projection on the disk, both these measurements are model-dependent. Therefore, the computed velocities of the loop-growth, given in Paper I, differ slightly, and those in Paper III very significantly, from the values given in Moore $e t$ al. However, we will still use Moore $e$ t al.'s Figures 8.4 and 8.5 for all further considerations, because they yield the most homogeneous set of measurements from the point of view of a comparison of both the cool and hot loops.

Let us demonstrate this by using Figure 4 which shows the positions of measured cool $(\mathrm{H} \alpha)$ loops at $14: 05$, shortly after the loops had become clearly visible, and at $16: 55$, when the first X-ray picture of the hot loops was made. In the Moore et al.'s figures Martin gave the average altitude of all $\mathrm{H} \alpha$ loops seen at a given time, measured from an assumed neutral $\left(H_{\|}=0\right)$ line running straight between the two bright ribbons. In Paper III, however, she gives the upper limit to the heights of the $\mathrm{H} \alpha$ loops near the southern end of the flare, measured from the apparent neutral line in the $\mathrm{H} \alpha$ pictures. It is obvious from Figure 4 that the altitudes in Paper III must result in significantly higher values than those in Moore et al. However, only the first method is comparable with the Nolte's (X-ray) mode of measurements. There is no other way than to assume a straight $H_{\|}=0$ line and to take average altitudes in the X-rays (cf. Paper I). Therefore, Moore et al.'s Figures 8.4 and 8.5 will be used throughout.

It is of interest to compare the growth of the flare loops in the two events: 29 July 1973 (without spots in the region), and 21 May 1980 (with spots). In the 21 May flare, which was situated close to the disk center, the loop system was inclined towards the south by an angle $\alpha$ that was between $20^{\circ}$ and $45^{\circ}$ (Švestka et al., 1982). If we want to make the flare-loop altitudes on May 21 identical with those observed on $29 \mathrm{July}$, we need a smaller $\alpha=10.5 \pm 2.0^{\circ}$. Thus the loops in the spotted region were definitely lower than those on 29 July: by a factor of 1.38 if $\alpha$ was $20^{\circ}$, and by 2.19 , if $\alpha$ was $45^{\circ}$.

\section{Temperature and Density}

Figure $8.3 \mathrm{~b}$ in Moore et al. shows the run of the effective temperature $T$ deduced from SOLRAD data. We have revised these values, because we now have new information on the detailed instrumental response of the SOLRAD 1-8 $\AA$ and 8-20 $\mathrm{A}$ channels, kindly provided to us by Don Horan of the Naval Research Laboratory, Washington, D.C. The revised temperatures, based on the flux ratio $F(1-8 \AA) / F(8-20 \AA)$, are plotted in Figure 5 for the first $2 \mathrm{hr}$ and 40 min development of the flare (filled circles), and representative $T$ values for later periods are given in Table I. These revised data yield significantly lower temperatures: $8.8 \times 10^{6} \mathrm{~K}$ instead of $12.6 \times 10^{6} \mathrm{~K}$ at $13: 40$, and $6.8 \times 10^{6} \mathrm{~K}$ instead of $9.0 \times 10^{6} \mathrm{~K}$ in Moore et al. at 17:00. Crosses in Figure 5 demonstrate the time variation of the emission measure EM corresponding to these revised temperatures.

Open circles and $x$ 's in Figure 5 show the temperature and emission measure values for the flare of 21 May 1980, observed by HXIS, as given by Švestka et al. (1982). These quantities refer to the brightest coarse element of the HXIS field of view $\left(32^{\prime \prime} \times 32^{\prime \prime}\right.$; this corresponds approximately to the area within the $0.25 C_{\mathrm{Max}}$ contour of the $\mathrm{X}$-ray flare). 


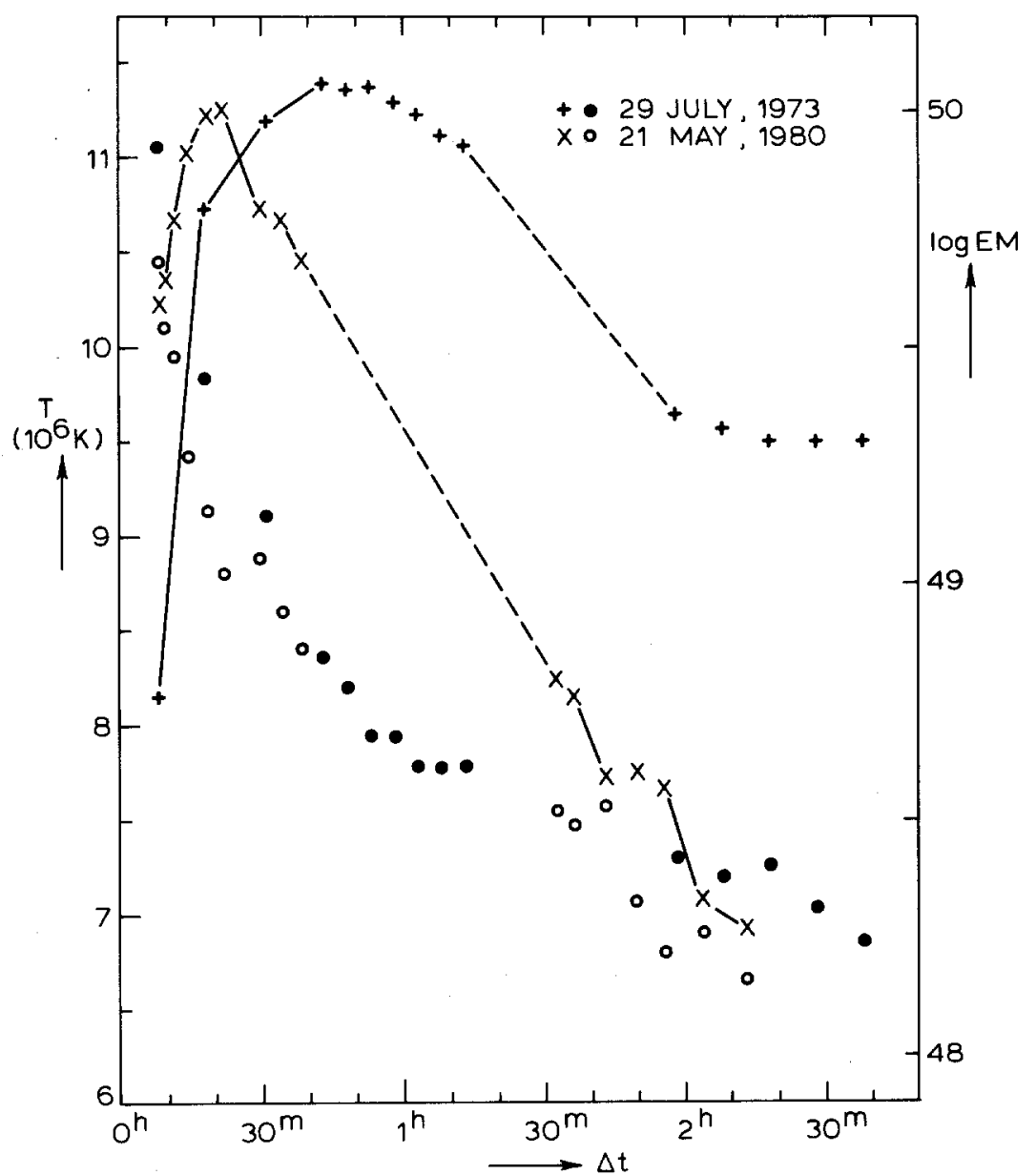

Fig. 5. Temperature (dots and circles) and emission measure (crosses and $x$ 's) in the two-ribbon flares of 29 July 1973 (in an old spotless region) and 21 May 1980 (in a developed sunspot group). The data come from flux ratios $F(1-8 \AA) / F(8-20 \AA)$ in the $29 \mathrm{July}$ flare, and $F(3.5-5.5 \mathrm{keV}) / F(5.5-8.0 \mathrm{keV})$ in the 21 May event. $\Delta \mathrm{t}$ is the time elapsed from the flare onset.

For the whole flare $T$ would be slightly lower and EM higher; for example, at $21: 31: 55$ (38.5 min after the flare onset) we get, for the whole coarse field of view, $T=8.3 \times 10^{6} \mathrm{~K}$ instead of $8.4 \times 10^{6} \mathrm{~K}$, and $\log E M$ by +0.4 higher than the value given in Figure 5 . These differences only strengthen the conclusions that we draw later from these curves.

The temperature and emission measure in the 21 May 1980 flare were deduced from the flux ratio $F(3.5-5.5 \mathrm{keV}) / F(5.5-8.0 \mathrm{keV})$ which covers the wavelength range from $1.55 \AA$ through $3.54 \AA$, i.e. much harder region of the spectrum than the SOLRAD data. One expects that higher-energy emission comes from a smaller flare region with higher temperature. This, e.g., is confirmed when comparing the Skylab temperatures of the 29 July flare in Paper II with the SOLRAD temperatures given here. At 16:40, the Skylab effective temperature, determined from the flux ratio $F(2-17 \AA) / F(2-54 \AA)$, was $4.5 \times 10^{6} \mathrm{~K}$, whereas $F(1-8 \AA) / F(8-20 \AA)$ on SOLRAD yielded $T \simeq 7.0 \times 10^{6} \mathrm{~K}$. 
Thus, the fact that the temperatures of both flares in Figure 5 are about the same indicates that $T$ in the spotted flare of 21 May was generally lower than $T$ in the spotless flare of $29 \mathrm{July}$. Note that about $2 \mathrm{hr}$ after the flare onset, when $T$ 's were still very much the same, the $E M$ in the 21 May flare was significantly smaller. One can anticipate, therefore, that a much larger volume was at lower temperatures at that time. The real difference in the temperatures remains unknown, because we do not have any observations of these flares in overlapping spectral regions.

Figure 8.6 in Moore et al. gives the electron density in the (post-) flare loops deduced from the cooling time under the assumption of pure radiative cooling. With the revised temperatures in Figure 5 also the densities change, and these new density values are given in Figure 6. The upper limit corresponds to cooling times deduced from a comparison of the altitudes of the hot and cool loops, whereas the lower limit is based on the separation and width of the $\mathrm{H} \alpha$ flare ribbons in Figure 3 (cf. Section 8.3.5 in Moore et al.). The resulting densities $n_{e}$ are now significantly lower than in Moore $e t$ al.: e.g., $9.3 \times 10^{9}-1.9 \times 10^{10} \mathrm{~cm}^{-3}$ instead of $2.1 \times 10^{10}-4.3 \times 10^{10} \mathrm{~cm}^{-3}$ at $14: 00,48 \mathrm{~min}$ after the flare onset.

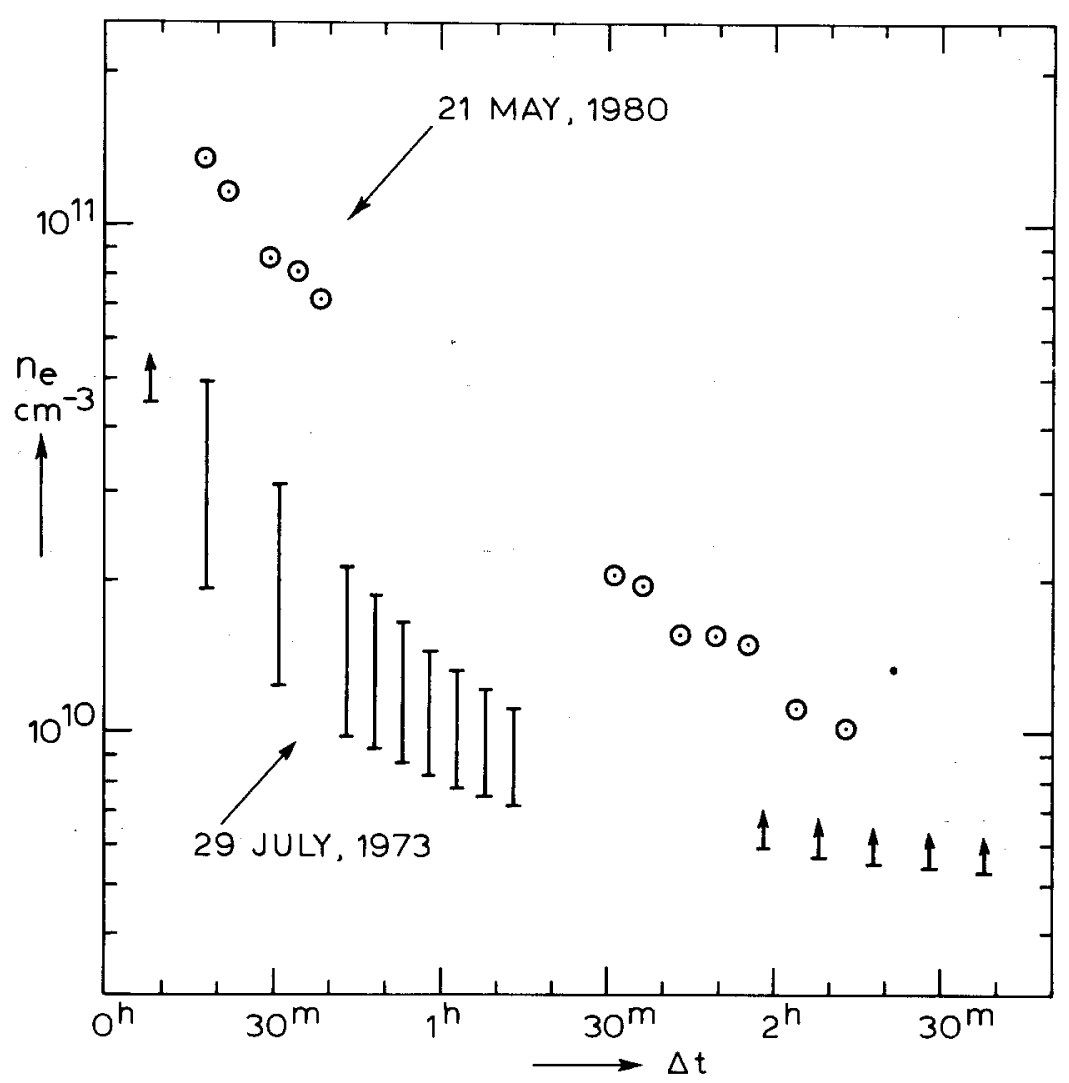

Fig. 6. Electron density in the hot (X-ray) loops of the two-ribbon flares of 29 July 1973 and 21 May 1980. See text for a more detailed explanation. 
We can compare again the densities in this spotless flare with those deduced for the 21 May event. The 21 May densities were estimated from the emission measure per coarse element assuming the flare thickness along the line of sight to be equal to the projected altitude of the loops on the solar disk. We get practically the minimum density values in the 21 May flare (Švestka et al., 1982). Thus Figure 6 shows that the densities in this flare were definitely higher than in the spotless flare of 29 July.

This is in agreement with the maximum emission measure being about the same in both the flares (Figure 5), whereas one would expect a smaller emitting volume producing the high-energy (1.55-3.54 $\AA$ ) emission in the 21 May flare. It also agrees with the observation that the $\mathrm{H} \alpha$ 'post-flare' loops were much better visible in the spotted flare, and even appeared, for some period of time, in emission. In order to appear in emission, the collisional excitation rate in the $\mathrm{H} \alpha$ line must exceed the radiative excitation rate, and this requires enhanced density. According to Zirin (private communication) one needs $n_{e} \simeq 10^{12}-10^{13} \mathrm{~cm}^{-3}$ to make the loops change from absorption to emission. The first emission loops were seen at $21: 07$ and were very bright at $21: 12$, i.e. $19 \mathrm{~min}$ after the flare onset (D. M. Rust, private communication). Assuming radiative cooling in these loops, $n_{e}=10^{12} \mathrm{~cm}^{-3}$ (lower Zirin's limit) and $T=12 \times 10^{6} \mathrm{~K}$, the cooling time is $\sim 125 \mathrm{~s}$. Thus these loops should have been hot and visible in X-rays 17 min after the flare onset, when our deduced density was $\sim 2 \times 10^{11} \mathrm{~cm}^{-3}$. This indicates, indeed, that the densities in the 21 May flare have been underestimated in Figure 6.

We may thus summarize all the results that arise from Figures 5 and 6 as follows:

The two-ribbon flare of 29 July 1973 which occurred in an old decaying spotless region produced higher loops with lower density than the flare of 21 May 1980 in which the ribbons were embedded in a sunspot group. This is consistent with the fact that a smaller volume is associated with the same temperature in the flare of 21 May than in $29 \mathrm{July}$. Generally, it seems that the loops in the spotless flare were hotter than those on 21 May; alternately, they might be equally hot at the time of their formation, but cooled slower. As Figure 5 shows, the spotted flare was decaying faster than the 29 July flare. This is confirmed also by the observation at $3: 20$, about $6.5 \mathrm{hr}$ after the flare onset, when HXIS could not detect any visible remnants of the flare loops (SMM did not look at the flare for a few hours before); this implies that the effective temperature of the loops, determined from $F(3.5-5.5 \mathrm{keV}) / F\left(5.5-8.0 \mathrm{keV}\right.$ ), was below $4.9 \times 10^{6} \mathrm{~K}$ if $E M$ was $10^{47} \mathrm{~cm}^{-3}$ (i.e. linear $E M=1.4 \times 10^{28} \mathrm{~cm}^{-5}$ ), or below $6.0 \times 10^{6} \mathrm{~K}$ for $E M=3 \times 10^{46} \mathrm{~cm}^{-3}$ (i.e. linear $\left.E M=4.2 \times 10^{27} \cdot \mathrm{cm}^{-5}\right)$. In contrast to that, the $F(1-8 \AA) / F(8-20 \AA)$ effective temperature in the flare of 29 July was still $5.7 \times 10^{6} \mathrm{~K}$ more than $11 \mathrm{hr}$ after the flare onset (cf. Table I). Skylab observations at that time yielded for the loop tops $T=4.4 \times 10^{6} \mathrm{~K}$, with linear $E M=8.7 \times 10^{28} \mathrm{~cm}^{-5}$, from the $F(2-17 \AA) / F(2-54 \AA)$ ratio (Paper II).

Thus, in conclusion, the loops of the spotless two-ribbon flare were higher, hotter, less dense, and the whole loop system was decaying much slower than in the spotted flare. 


\section{The $H \alpha$ Ribbons in Relation to the X-Ray Loops}

Figure 8.10 in Moore et al. and Figure 7 in the present paper show the isophotes of the $\mathrm{H} \alpha$ bright ribbons at $16: 32$ on July 29, 1973, as measured at the McMath-Hulbert Observatory. The isophotes correspond to measured intensity in units of local $\mathrm{H} \alpha$ background equal to $1.04,1.14,1.23,1.33$, and 1.44 .

By using measurements from the SECASI, one adopts 2.5 for the ratio between the continuum and the $\mathrm{H} \alpha$ background at the center of the solar disk. The undisturbed local $\mathrm{H} \alpha$ background centerward of the flare was 0.85 of the $\mathrm{H} \alpha$ background at the center of the disk. Thus the intensities in units of the central continuum are obtained from the measured intensities by multiplication by $0.85 / 2.5=0.34$ : for the five isophotes we thus get $0.35,0.39,0.42,0.45$, and 0.49 units of the central continuum.

Figure 8.7 in Moore et al. shows the isophotes of the X-ray flare at 16:43, but on a slightly different scale from Figure 8.10. We have brought both these plots of isophotes to the same scale and superposed the $\mathrm{X}$-rays on the $\mathrm{H} \alpha$ picture in our Figure 7 . The $\mathrm{X}$-ray isophotes represent 5.4, 10.7, 21.4, and $42.9 \times 10^{5} \mathrm{erg} \mathrm{cm} \mathrm{s}^{-1}(\operatorname{arc~sec})^{-2}$ received at Earth orbit (cf. Figure 2 in Paper II; kindly note that the 1 arc min scale in that figure is incorrect).

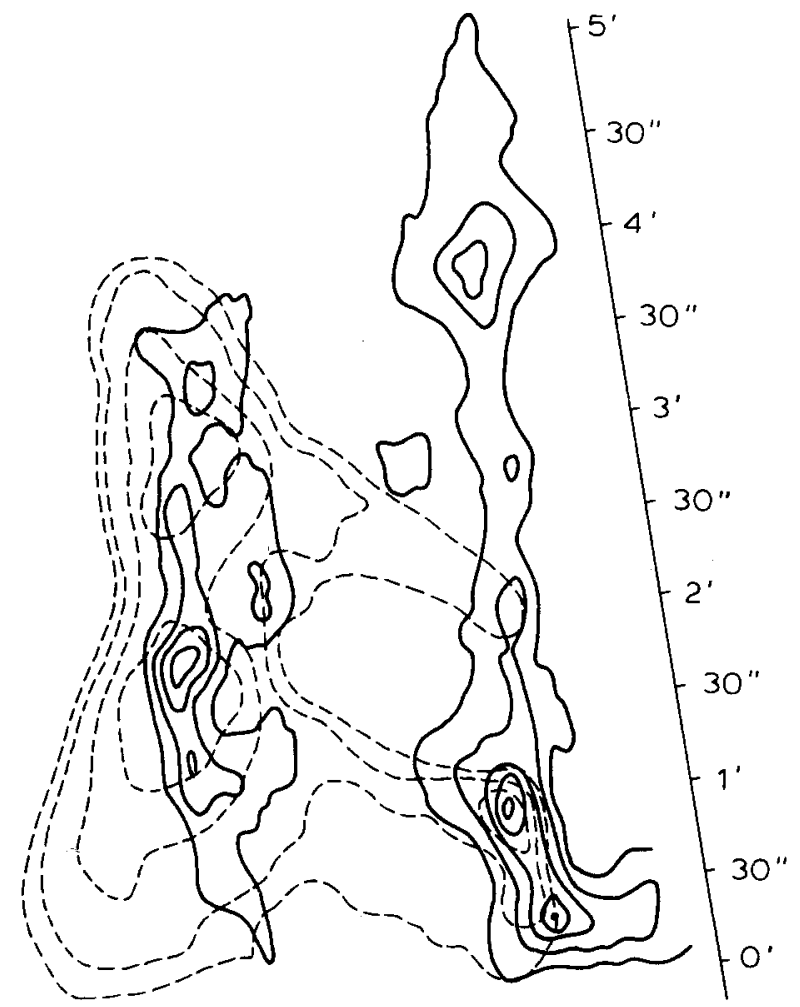

Fig. 7. Overlay of the soft X-ray image (16:43) over the $\mathbf{H} \alpha$ ribbons $(16: 32)$. X-ray isophotes: $5.4,10.7$, 21.4, and $42.9 \times 10^{-5} \mathrm{erg} \mathrm{cm}^{-2} \mathrm{~s}^{-1}(\operatorname{arc~sec})^{-2}$ at the Earth (dashed lines); $\mathrm{H} \alpha$ isophotes: 0.35, 0.39, 0.42, 0.45 , and 0.49 units of continuum near $\mathrm{H} \alpha$ in the center of the Sun (full lines). 
One can see from Figure 7 that the highest condensations in the $\mathrm{H} \alpha$ bright ribbons align with the footpoints of the brightest loops in the soft X-ray image. We have been able to show further (in Figure 8.9 in Moore et al., and in Figure 4 of Paper II) that the enhanced X-ray brightness is a result of increased linear emission measure, whereas the temperature is about the same throughout all the loops. Thus the brightest patches in the $\mathrm{H} \alpha$ ribbons are apparently the footpoints of the X-ray loops in which the density is most enhanced.

This result can be interpreted in two different ways: either the increased brightness is caused by enhanced plasma density in the loops $\left(N_{e}\right.$ is increased in $\left.E M_{l}=N_{e}^{2} l\right)$, or it is produced by an increased number of elementary loops along the line of sight $(l$ is increased in $E M_{l}=N_{e}^{2} l$ ). In this late phase of the flare new loops are still expected to be formed, but the occurrence of new formations must be infrequent and their lifetime (cooling time) long. Thus we have a quasi-thermal situation, and the energy transfer from the tops of the hot coronal loops to the chromosphere should be predominantly by means of heat conduction. But heat conduction is almost independent of density. Thus it appears that the second alternative is more likely: the $\mathrm{H} \alpha$ ribbons show enhancements wherever the spatial density of individual coronal flare loops is increased; because of limited resolution we integrate the contribution of the individual loops to the chromospheric heating.

\section{Acknowledgements}

We want to thank Drs D. M. Rust, A. Schadee, and H. Zirin for stimulating discussions. The analysis of the Skylab data was supported by NASA Contracts NAS 8-27758 (J.T.N. and R.D.P.) and NAS 8-32984 (Z.S.). The HXIS observations and their analysis was made possible by the support of the Netherlands Committee for Geophysics and Space Research (GROC) of the Royal Netherlands Academy of Arts and Sciences (KNAW).

\section{References}

Bruzek, A.: 1959, Z. Astrophys. 31, 99.

Howard, R. and Švestka, Z.: 1977, Solar Phys. 54, 65.

Hoyng, P., Duijveman, A., Machado, M. E., Rust, D. M., Švestka, Z., and 6 co-authors: 1981, Astrophys. $J$. Letters 246, L-155.

Kahler, S. W., Hildner, E., and Van Hollebeke, M. A. I.: 1978, Solar Phys. 57, 429.

Martin, S. F.: 1979, Solar Phys. 64, 165 (Paper III).

Michalitsanos, A. G. and Kupferman, P.: 1974, Solar Phys. 36, 403.

Moore, R. L. and LaBonte, B. J.: 1980, in M. Dryer and E. Tandberg-Hanssen (eds.), 'Solar and Interplanetary Dynamics', IAU Symp. 91, 207.

Moore, R. L., McKenzie, D. L., Švestka, Z., Widing, K. G. and 12 co-authors: 1979, in P. A. Sturrock (ed.), Solar Flares, Proceedings of the Second Skylab Workshop, Colorado Associated University Press, p. 341.

Munro, R. H., Gosling, J. T., Hildner, E., MacQueen, R. M., Poland, A. I., and Ross, C. L.: 1979, Solar Phys. 61, 201.

Nolte, J. T., Gerassimenko, M., Krieger, A. S., Petrasso, R. D., and Švestka, Z.: 1979, Solar Phys. 62, 123 (Paper I). 
Perron, C., Domingo, V., Reinhard, R., and Wenzel, K. P.: 1978, J. Geophys. Res. 83, 2017.

Petrasso, R. D., Nolte, J. T., Gerassimenko, M., Krieger, A. S., Krogstad, R., Seguin, F. H., and Švestka, Z.: Solar Phys. 62, 133 (Paper II).

Priest, E. and Milne, A. M.: 1980, Solar Phys. 65, 315.

Rust, D. M., Nakagawa, Y., and Neupert, W. M.: 1975, Solar Phys. 41, 397.

Sturrock, P. A. (ed.): 1979, Solar Flares, Proceedings of the Second Skylab Workshop, Colorado Associated University Press.

Švestka, Z.: 1976, Solar Flares, D. Reidel Publ. Co., Dordrecht, Holland.

Švestka, Z., Stewart, R. T., Hoyng, P., Van Tend, W., Acton, L. W., Gabriel, A. H., Rapley, C. G., and 7 co-authors: 1982, Solar Phys. 75, 305.

Van Beek, H. F., Hoyng, P., Lafleur, H., and Simnett, G. M.: 1980, Solar Phys. 65, 39. 